\title{
Reward and Risk for Opportunistic Spectrum Accessing in Cognitive Radio Networks
}

\author{
Miao Pan*, Yang Song*, Pan $\mathrm{Li}^{\dagger}$ and Yuguang Fang* \\ *Department of Electrical and Computer Engineering, University of Florida, Gainesville, FL 32611 \\ ${ }^{\dagger}$ Department of Electrical and Computer Engineering, Mississippi State University, Mississippi State, MS 39762 \\ Email: \{miaopan@, yangsong@, fang@ece.\}ufl.edu, li@ece.msstate.edu
}

\begin{abstract}
Cognitive Radio technology releases the spectrum from shackles of authorized licenses and facilitates the trading of spectrum bands. In the spectrum market, primary service providers (PSPs) set price for the vacant licensed bands of primary users (PUs) and sell them for monetary gains, and the secondary service provider (SSP) can buy the bands and opportunistically use them to satisfy the service demands of secondary users (SUs) when the primary services are not active. However, when there are multiple bands available, the SSP confronts the challenges of how to choose bands and how to split the overall traffic on them considering both his monetary reward and the potential risk, from the unpredictable activities of the primary services, for opportunistic spectrum accessing (OSA). In this paper, we propose a reward and risk based band-mix selection algorithm to address these concerns of the SSP, and help the SSP to make appropriate decisions of traffic splitting over available spectrum band-mix, consisting of both the band belonging to SSP itself and the bands from PSPs. By numerical simulations, we verify our theoretical analysis and show that the proposed spectrum band-mix selection effectively improves the spectrum utilization as well as the satisfactory degree of SUs.
\end{abstract}

Index Terms-Traffic Splitting, Expected Reward, Risk, BandMix Selection

\section{INTRODUCTION}

Recent years have witnessed the booming growth of wireless networks and flourish of various wireless services. In parallel with that, current static spectrum allocation policy of Federal Communications Commission (FCC) [1] results in the exhaustion of available spectrum, while many licensed spectrum bands are extremely under-utilized. As one of the most promising solutions to improve spectrum utilization, cognitive radio technology allows the secondary users (SUs) to opportunistically access to vacant bands belonging to primary users (PUs) in either temporal or spatial domain.

The idea of opening up the licensed spectrum bands in cognitive radio networks initiates the market of spectrum trading, and promotes a batch of interesting research on related topics. Specifically, in [2], Grandblaise et al. generally describe the possible scenarios and introduce some microeconomics inspired mechanisms for opportunistic spectrum accessing (OSA), and in [3], Sengupta and Chatterjee propose an economic framework for OSA and service pricing to guide the design of dynamic spectrum allocation algorithms as well as service pricing mechanisms that the service providers can possibly use. From the view of system design, models in game theory, by Wang et al. in [4] and Pan et al. in [5], and auction

This work was supported in part by the U.S. National Science Foundation under grants CNS-0721744 and CNS-0916391. designs in microeconomics, by Zhou et al. in [6] and Jia et al. in [7], are exploited to construct the spectrum trading mechanisms with desired properties, such as Pareto efficiency, incentive compatibility, power efficiency, etc.. From the view of the primary service provider (PSP), Xing et al. in [8] and Niyato et al. in [9] have well investigated the spectrum pricing issues in the spectrum market, where multiple PSPs, whose goal is to maximize the monetary gains with their vacant spectrum, compete with each other to offer spectrum access to the secondary service provider (SSP). From the view of the SSP, people are interested in how the SSP optimally distributes SUs' traffic demands over the spectrum bands when there is more than one unoccupied licensed band. Motamedi and Bahai in [10] has formulated this problem as a multi-armed bandit problem and proposes a reinforce learning algorithm to consistently track the best band in terms of band conditions.

However, the concept of the best band for OSA tends to be ambiguous considering the characteristics of different bands. As described in [8], [9], the spectrum bands from PSPs may be evaluated unequally by the SSP from different perspectives, such as the frequency of the available band, the segment type of the band (i.e., contiguous segment or discontinuous one), the price of the band, the location of the PSP, etc.. Among all these factors, the PUs' activities on the band are the most crucial one for the SSP, because the unexpected returning of primary services may terminate the SSP's spectrum provision to the SUs and incur enormous monetary risk for the SSP on its spectrum purchasing. Besides, heaping all the traffic on one band makes the secondary services vulnerable to the activities of the primary services.

In this paper, we propose to leverage SSP's expected reward to model the SSP's concern from economic aspect and the variance of this reward to represent the potential risk for OSA incurred by primary services' coming back. Instead of swarming all the traffic over one band, we put "eggs" into different "baskets" [11], i.e., we make the SSP split all the traffic on a mix of bands. The reason is that it is generally impossible for bands with different frequencies to do poorly at the same time. In particular, we illustrate how the SSP optimally splits the traffic over the band-mix of risk-free band of his own and risky bands from PSPs, and how the SSP selects the proportional composition of the risky band-mix from the PSPs. By numerical simulations, we not only verify the theoretical results but also show that the proposed spectrum band-mix selection is effective in improving the spectrum utilization and SUs' satisfaction degree. 


\section{SySTEM MODEL}

\section{A. Spectrum Market}

We consider a spectrum market in cognitive radio networks [9] with multiple PSPs operating on $n$ different spectrum bands and a SSP who serves a group of SUs as shown in Fig. 1. The SUs can take opportunistic use of these licensed spectrum bands when the primary services are not on, but must evacuate from these bands immediately when primary services become active. In addition, we assume all the spectrum transactions take place at starting time of each period ${ }^{1}$, and the payment for spectrum trading is non-refundable.

In this case, PSPs will set reasonable prices for the unoccupied bands considering the quality of the bands as well as competition among the PSPs in the spectrum market [7][9], and sell those bands periodically for monetary gains. Correspondingly, if the SSP (e.g., the base station (BS) or the access point (AP)) is short of radio resources for the traffic demands of the SUs (Suppose there is a upper bound for SUs' traffic demands that the SSP can satisfy with his own band. In this paper, the limit is assumed to be $k(0 \leq k \leq 1)$ portion of overall traffic), the SSP will play the role of trading agent for the SUs [3]. Specifically, the SSP will try to select a mix of currently vacant licensed bands ${ }^{2}$, buy those bands from the PSPs and charge the SUs with the prices set by PSPs. Meanwhile, the SSP is in charge of the transmissions on the bands purchased from PSPs as well as his own band, and share these radio resources among multiple SUs in a time-division multiple access (TDMA) manner.

\section{B. Reward and Risk for OSA}

Suppose the reward for the SSP is $R_{s}$, if the SSP distribute one Mbps traffic of secondary services over his own band for a selling/buying time period. As to the SSP, $R_{s}$ should be a fixed number because the reward can be guaranteed if the SSP satisfies the traffic demands of SUs with his own band. Since $R_{s}$ is the reward attained by the SSP through using his own spectrum band, and the SSP does not worry about the potential risk brought by primary services, $R_{s}$ can be regarded as riskfree reward. On the other hand, if the SSP is using band $i$ bought from PSPs, his monetary gains for one time period can be denoted as $R_{i}$, where $R_{i}$ is a random variable with mean $E\left(R_{i}\right)$ representing the expected reward of the $\mathrm{SSP}^{3}$, and variance $\sigma_{i}^{2}$ representing the risk for OSA due to the coming back of primary services on band $i$.

\footnotetext{
${ }^{1}$ The selling/buying period should not be too long (e.g., months or years) to make dynamic spectrum access infeasible, and it should not be too short (e.g., seconds or minutes) to incur overwhelming overhead in spectrum trading. The typical duration is hours or days as shown in [12]. In the rest of paper, we assume that all the spectrum transactions are of fixed duration, so that the time parameter is not included in our formulation.

${ }^{2}$ Actually, the process of selecting the band-mix for OSA may be divided into two stages. The first stage starts with sampling and observation, and ends with statistical values about the future performances of available spectrum bands. Yin et al. in [13] has accomplished the first stage work of bandmix selection for OSA. They carried out a set of spectrum measurements in the $20 \mathrm{MHz}$ to $3 \mathrm{GHz}$ spectrum bands at 4 locations concurrently in Guangdong province of China, and conducted a set of detailed analysis of the first and second order statistics of the collected data, including channel occupancy/vacancy statistics, channel utilization, also spectral and spatial correlation of these measures; The second stage starts with the relevant values about future performances, and ends with the selection of the band-mix. In this paper, we are only concerned with the second stage.

${ }^{3}$ We assume $E\left(R_{i}\right) \geq R_{s}$ in the rest of paper.
}

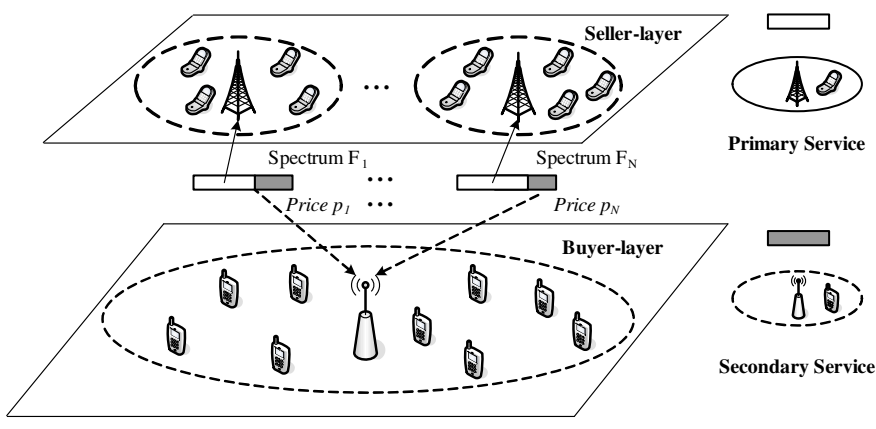

Fig. 1. Spectrum trading system

As depicted in the system architecture of Fig. 1, the SSP can opportunistically use multiple spectrum bands according to geographical constraints. We assume the SSP has $N=$ $\{1,2, \cdots, n\}$ spectrum bands owned by PSPs to select from, and the corresponding reward is $\left\{R_{1}, R_{2}, \cdots, R_{n}\right\}$. Given the characteristics of the candidate spectrum bands, the SSP needs to decide how to split the traffic on all the bands including both risk-free band of his own and risky bands purchased from PSPs. Suppose the overall traffic of the SUs is normalized to 1 , the problem above can be formulated as the follows

$$
\left\{\begin{array}{l}
R=(1-\eta) R_{s}+\eta R_{p} \\
R_{p}=\sum_{i=1}^{n} \omega_{i} R_{i}
\end{array}\right.
$$

where $R$ is the total reward for the SSP, $R_{s}$ is the risk-free reward by using the SSP's own band, and $R_{p}$ is the risky reward for splitting traffic on the band-mix of PSPs' bands. The value of $\eta \in[0,1)$ indicates that the proportion of traffic split on the band-mix of PSPs' bands, while $(1-\eta)$ is the rest of traffic split on the SSP's own band. $R_{p}$ is a weighted sum of all possible $R_{i}$ for the SSP with $\omega_{i}$ as non-negative weights, where $\omega_{i}$ represents the proportional composition of the risky band-mix, and $\sum_{i=1}^{n} \omega_{i}=1$. The expected value and the variance of $R_{p}$ can be written as

$$
\left\{\begin{array}{l}
E\left(R_{p}\right)=E\left(\sum_{i=1}^{n} \omega_{i} R_{i}\right) \\
\sigma_{R_{p}}^{2}=\sum_{i=1}^{n} \omega_{i}^{2} \sigma_{i}^{2}+2 \sum_{i=1}^{n} \sum_{j \neq i}^{n} \omega_{i} \omega_{j} \sigma_{i} \sigma_{j} \rho_{i j} .
\end{array}\right.
$$

where $\sigma_{i j}=\sigma_{i} \sigma_{j} \rho_{i j}$ is the covariance between $R_{i}$ and $R_{j}$, and $\rho_{i j}\left(-1 \leq \rho_{i j} \leq 1\right)$ is the correlation coefficient.

From Equation (1) and (2), the SSP's expected reward and the risk for OSA can be denoted as

$$
\left\{\begin{array}{l}
E(R)=(1-\eta) R_{s}+\eta E\left(R_{p}\right) \\
\sigma_{R}^{2}=\eta^{2} \sigma_{R_{p}}^{2} .
\end{array}\right.
$$

\section{Concepts Related to the Band-Mix Selection for OSA}

1) Band-Mix Selection Rule: Intuitively, the SSP is able to maximize $E(R)$ by pouring all the traffic of SUs over a particular band with the maximal expected reward. However, the risk of using that band may be quite high. A rational or risk-averse SSP is not likely to gamble all the traffic on one band since the reward may be extraordinarily low considering the activities of primary services. Therefore, we propose the band-mix selection criterion for OSA as the follows.

Reward-Risk (Dual-R) Rule: The SSP should select a band-mix consisting of risk-free band and risky bands with maximum expected reward $(E(R))$ for given risk $\left(\sigma_{R}\right)$ for OSA, or a band-mix with minimum risk $\left(\sigma_{R}\right)$ for given expected reward $(E(R))$ for OSA. 
2) Feasible Selection Set and Candidate Selection Frontier: The feasible selection set (FSS) for the SSP represents all band-mixes that can be constructed from a given set of spectrum bands. Adhering to Dual-R rule, the candidate selection frontier (CSF) is defined to be the set of all the band-mixes with the reward-risk pairs in FSS which are not dominated by any other band-mix in the FSS. Given a fixed risk, the corresponding band-mix on the CSF always provides the highest reward. Or, given a fixed expected reward, the corresponding band-mix on the CSF always provides the lowest risk for OSA.

3) SSP's Utility Function: The consequences of the bandmix selection and traffic splitting correspond to the SSP receiving a certain amount of reward. The utility function, i.e., $U(R)$, simultaneously measures the SSP's satisfaction of receiving that amount of reward and the risk-aversion associated with that amount. In this paper, the SSP's utility function is a increasing and concave down function, which may be polynomial, exponential, etc. A rational SSP's goal is to find the optimal traffic splitting over the band-mix of riskfree band and risky bands to maximize the expected utility, i.e., $E(U(R))$, with respect to all expected reward-risk pairs of possible band-mixes for OSA.

Considering the reward and risk for OSA, the SSP with the knowledge of $R_{s}$ and $R_{i}$ has to decide how to split the traffic between risky bands and risk-free band (i.e., $\eta$ ) and determine the proportional composition of the risky band-mix from the PSPs (i.e., $\omega_{i}$ ).

\section{BAND-MIX SELECTION FOR OSA}

\section{A. Traffic Splitting over Risk-free and Risky Bands}

With the established knowledge about the characteristics of the band-mix, let us recap the relation between the SSP's traffic splitting over any arbitrary band-mix including the SSP's own band as well as the bands from PSPs, the SSP's expected reward, and the risk for OSA as shown in Equation (3). After eliminating $\eta$, we find

$$
\left\{\begin{array}{l}
E(R)=R_{s}+\theta \sigma_{R_{p}} \\
\theta=\left(E\left(R_{p}\right)-E\left(R_{s}\right)\right) / \sigma_{R_{p}}
\end{array}\right.
$$

In terms of any arbitrarily selected band-mix, therefore, the SSP's expected reward for his traffic splitting over these bands is linearly related to $\sigma_{R_{p}}$, i.e., the risk for opportunistic accessing to the risky band-mix from PSPs, and the intercept of this linear function is the risk-free reward $R_{s}$. Given any selected mix of bands from PSPs, the slope of Equation (4), $\theta$, is determined by the parameters $E\left(R_{p}\right)$ and $\sigma_{R_{p}}$ of the particular risky band-mix from PSPs being considered. Thus, we obtain results as the follows

- By a suitable choice of $\eta$ satisfying the constraints of the SSP's traffic delivering on his risk-free band $(0 \leq$ $(1-\eta) \leq k)$, the SSP can use any mix of spectrum bands to obtain an expected reward, $E(R)$ in the range of $\left[k R_{s}+(1-k) E\left(R_{p}\right), E\left(R_{p}\right)\right)$.

- As the SSP increases the expected reward by enlarging the amount of the traffic split on the risky band-mix from PSPs, the standard deviation $\sigma_{R}$ of the reward for OSA also becomes proportionately greater.

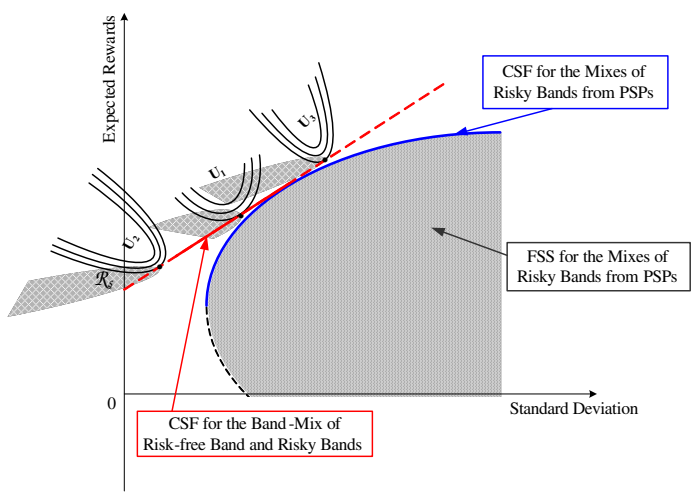

Fig. 2. The geometrical illustration of the band-mix selection for OSA

- Both the FSS and CSF are along the line starting at the point with $\left((1-k) \sigma_{R_{p}}, k R_{s}+(1-k) E\left(R_{p}\right)\right)$ and ending at the point with given $\left(\sigma_{R_{p}}, E\left(R_{p}\right)\right)$ on the Expected Reward - Standard Deviation plane.

Now consider all possible mixes of risk-free band from SSP and risky bands from PSPs. Those band-mixes have the same $\theta$ value will lie on the same line, but those have different $\theta$ values will produce different lines between reward and risk for OSA. All the possible lines construct the FSS. The SSP has to decide the line of CSF (i.e., the proper value of $\theta$ ), and how intensively to split his traffic between the SSP's band and PSPs' bands along CSF (i.e., the proper value of $\eta$ ).

To find the CSF for the traffic splitting, the SSP adhering to the proposed Dual-R rule seeks to minimize the variance of his overall reward, $\sigma_{R}^{2}$, associated with any expected reward he may choose, $E(R)$, by confining all his traffic splitting to the band-mix with the largest $\theta$ value. Note that only partial of the line with maximum $\theta$ is CSF with regards to the constraints of the risk-free band of the SSP, which is $0 \leq(1-\eta) \leq k$.

After determining the CSF by maximizing $\theta$, the SSP can complete his selection for the band-mix of risky bands owned by PSPs by substituting the maximized $\theta$ in Equation (4). Furthermore, referring to his utility function, the SSP is also able to decide the the optimal amount of traffic split on the risky band-mix from the PSPs as well as the optimal amount of traffic on the SSP's own risk-free bands (i.e., the unique best value of $\eta$ ). The algebraic derivations given above can be represented graphically as in Fig. 2.

\section{B. The Optimal Proportional Composition of the Risky Band- Mix}

Although we finish the band-mix selection of risky bands geometrically, it is still necessary to quantify the proportional composition of selected risky band-mix (i.e., to determine the proper value of $\omega_{i}$ ) and dig deeper in the properties of the selected risky band-mix from PSPs.

From Equation (1), the reward for opportunistically using risky band-mix from the PSPs can also be expressed as

$$
\begin{aligned}
R_{p} & =\sum_{i=1}^{n}\left[\omega_{i}\left(R_{i}-R_{s}\right)+\omega_{i} R_{s}\right] \\
& =R_{s}+\sum_{i=1}^{n} \omega_{i}\left(R_{i}-R_{s}\right),
\end{aligned}
$$


where $\sum_{i=1}^{n} \omega_{i}=1$. Thus, the expected reward and risk for OSA on any risky band-mix from PSPs are consequently

$$
\left\{\begin{array}{l}
E\left(R_{p}\right)=R_{s}+\sum_{i=1}^{n} \omega_{i}\left(E\left(R_{i}\right)-R_{s}\right) \\
\sigma_{R_{p}}=\sum_{i=1}^{n} \sum_{j=1}^{n} \omega_{i} \omega_{j} \sigma_{i j}
\end{array}\right.
$$

We further simplify the notations in the group of equations above by defining

$$
\left\{\begin{array}{l}
X_{i}=E\left(R_{i}\right)-R_{s} \\
X=\sum_{i=1}^{n} \omega_{i} X_{i}
\end{array}\right.
$$

where $X_{i}$ is the excess reward.

Considering the definition in Equation (4), the value of $\theta$ can thus be written as ${ }^{4}$

$$
\theta=\frac{E\left(R_{p}\right)-R_{s}}{\sigma_{R_{p}}}=\frac{X}{\sigma_{X}}=\frac{\sum_{i=1}^{n} \omega_{i} X_{i}}{\left(\sum_{i=1}^{n} \sum_{j=1}^{n} \omega_{i} \omega_{j} \sigma_{X_{i j}}\right)^{1 / 2}} .
$$

As we have discussed in the last section, for the SSP, the optimal risky band-mix is the one that maximizes $\theta$ as shown in Equation (8). Note that the value of $\theta$ is unchanged by any proportionate change in all $\omega_{i}$, which means $\theta$ is not affected by any changes of $\eta$. So, to select the optimal band-mix of risky bands can be simplified into maximizing this value of $\theta$ subject to the constraint $\sum_{i} \omega_{i=1}^{n}=1$.

By examining the partial derivatives of $\theta$ with respect to the $\omega_{i}$, we attain

$$
\frac{\partial \theta}{\partial \omega_{i}}=\left(\sigma_{X}\right)^{-1}\left[X_{i}-\lambda\left(\omega_{i} \sigma_{X_{i i}}+\sum_{j=1, j \neq i}^{n} \omega_{j} \sigma_{X_{i j}}\right)\right],
$$

where

$$
\lambda=\frac{X}{\sigma_{X}^{2}}=\frac{\sum_{i=1}^{n} \omega_{i} X_{i}}{\sum_{i=1}^{n} \sum_{j=1}^{n} \omega_{i} \omega_{j} \sigma_{X_{i j}}} .
$$

The necessary and sufficient conditions on the relative values of the $\omega_{i}$ for the unique maximum $\theta$ are obtained by setting the derivatives above equal to zero, which gives

$$
X_{i}=\zeta_{i} \sigma_{X_{i i}}+\sum_{j=1, j \neq i}^{n} \zeta_{j} \sigma_{X_{i j}},
$$

where

$$
\zeta_{i}=\lambda \omega_{i} .
$$

Let $\vec{\zeta}=\left\{\zeta_{1}, \zeta_{2}, \cdots, \zeta_{n}\right\}$ and $\vec{X}=\left\{X_{1}, X_{2}, \cdots, X_{n}\right\}$. For $i=\{1,2, \cdots, n\}$, Equation (11) can be extended as

$$
\vec{\zeta} \operatorname{Cov}\left(X_{i}, X_{j}\right)=\vec{X} .
$$

It should be noted that the set of equations are linear in terms of the own-variances, $\sigma_{X_{i i}}$, the pooled covariances, $\sigma_{X_{i j}}$, and excess reward, $X_{i}$, of the risky bands from the PSPs. Since the covariance matrix, $\operatorname{Cov}\left(X_{i}, X_{j}\right)$, is positive definite and thus non-singular, the unique solution to this series of equations is a vector $\vec{\zeta}^{*}$ consisting of

$$
\zeta_{i}^{*}=\sum_{j=1}^{n} \sigma_{X_{i j}}^{(-1)} X_{j},
$$

\footnotetext{
${ }^{4} \mathrm{Here}, \sigma_{i j}$ is equal to $\sigma_{X_{i j}}$. See proof in the Appendix.
}

where $\sigma_{X_{i j}}^{(-1)}$ represents the $i j$-th element ${ }^{5}$ of the inverse of the covariance matrix, $\mathrm{Cov}^{(-1)}\left(X_{i}, X_{j}\right)$. This solution may also be expressed as

$$
\omega_{i}^{*}=\left(\lambda^{*}\right)^{-1} \sum_{j=1}^{n} \sigma_{i j}^{(-1)}\left(E\left(R_{j}\right)-R_{s}\right)
$$

where $\sigma_{i j}^{(-1)}$ represents the $i j$-th element of the inverse of the covariance matrix, $\operatorname{Cov}^{(-1)}\left(R_{i}, R_{j}\right)$ and $\omega_{i}^{*}$ is the corresponding proportional composition for risky band $i$ in the selected optimal risky band-mix from PSPs.

Moreover, $\zeta_{i}=\lambda \omega_{i}$ implies

$$
\sum_{i=1}^{n} \zeta_{i}=\lambda \sum_{i=1}^{n} \omega_{i}
$$

Leveraging the constraint $\sum_{i=1}^{n} \omega_{i}=1$, we find that $\lambda^{*}$ is

$$
\lambda^{*}=\lambda^{*} \sum_{i=1}^{n} \omega_{i}^{*}=\sum_{i=1}^{n} \zeta_{i}^{*}
$$

By substituting Equation (14) and (17) into Equation (15), the $\zeta_{i}^{*}$ obtained in Equation (14) can consequently lead to the solution of $\omega_{i}^{*}$, i.e., the optimal proportional composition of risky band-mix from the PSPs.

As for the optimal selection of risky band-mix, it is interesting to note that by substituting $\zeta_{i}^{*}=\lambda^{*} \omega_{i}^{*}$ into Equation (11), $\omega_{i}^{*}$ can be denoted as

$$
\left\{\begin{array}{l}
\omega_{i}^{*}=\frac{\lambda_{i}}{\lambda^{*}}-\sum_{j \neq i} \omega_{j}^{*} \frac{X_{i j}}{\sigma_{X_{i i}}} \\
\lambda_{i}=\frac{X_{i}}{\sigma_{X_{i i}}} .
\end{array}\right.
$$

Therefore, if the SSP selects the risky band-mix from PSPs on the assumption that all the covariances of the risky bands are zero, he can pick his optimal band-mix of risky bands very simply by determining $\lambda_{i}$, which is equal to $\frac{E\left(R_{i}\right)-R_{s}}{\sigma_{i}}$ according to Equation (18) and (7), and setting each $\omega_{i}^{*}=\lambda_{i} / \sum_{i} \lambda_{i}$, where $\sum_{i} \lambda_{i}=\lambda^{*}=X^{*} / \sigma_{X^{*}}^{2}$ for no covariances. With this simplified assumption, the value of $\lambda_{i}$ of each risky band is sufficient to determine the optimal band-mix from PSPs. In the more general case with non-zero covariances, a set of linear equations must be solved in the usual way, but neither linear nor non-linear programming is required, and there exists an unique solution for the SSP, which is one point on the CSF for the mixes of risky bands owned by PSPs.

Once the the proportional composition of the optimal risky band-mix, $\omega_{i}^{*}$, is determined, the corresponding expected reward $E\left(R_{p}^{*}\right)$ and risk $\sigma_{R_{p}^{*}}$ of the risky band-mix can be calculated. Furthermore, the SSP has the knowledge of $R_{s}$, so that the CSF for traffic splitting over risk-free and risky bands can also be determined. The only left issue for the SSP is to find a point on the CSF (i.e., to determine the proper value of $\eta)$ to maximize his expected utility according to his own utility function subject to the constraint that $0 \leq(1-\eta) \leq k$, which is not difficult to solve by following a standard optimization procedure.

${ }^{5}$ Please note the difference between $\sigma_{X_{i j}}^{(-1)}$ and $\left(\sigma_{X}\right)^{-1}$ in Equation (9). 


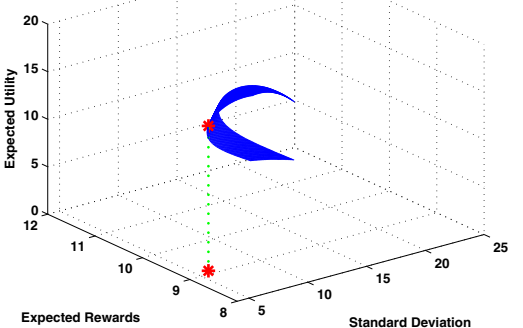

(a) Maximum utility with quadratic utility function

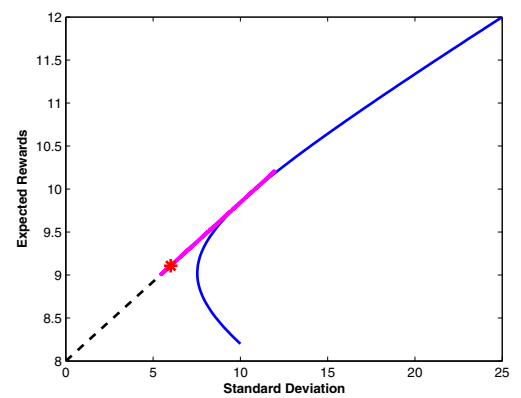

(d) The optimal band-mix selected with quadratic (e) utility function

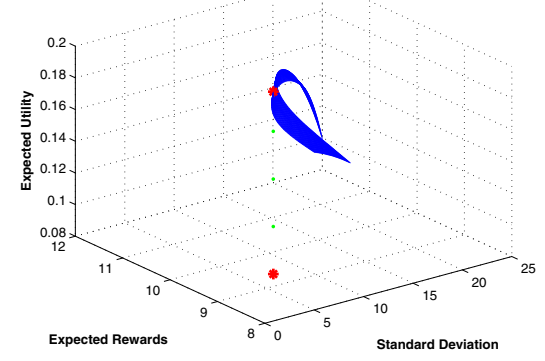

(b)



(e) The optimal band-mix selected with exponential utility function

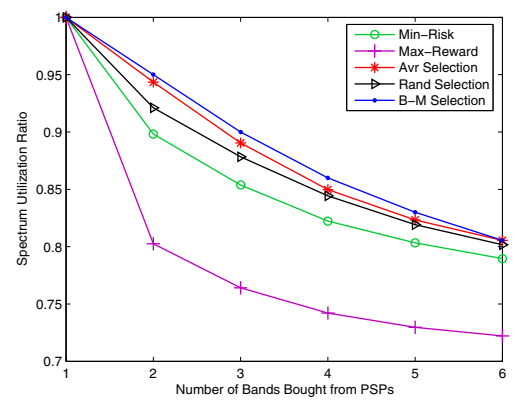

(c) Performance of different spectrum selection schemes in terms of spectrum utilization

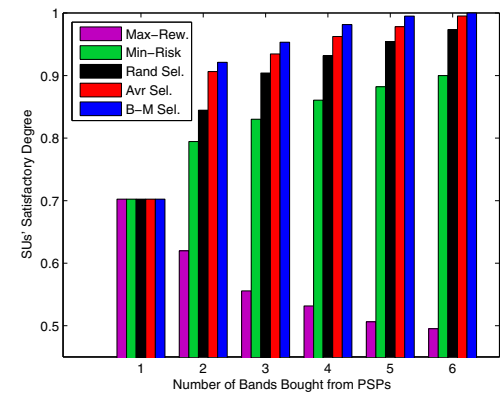

(f) Performance of different spectrum selection schemes in terms of secondary s' satisfactory degree

Fig. 3. Verification of the theoretical analysis and performance of the proposed band-mix selection

\section{Simulation AND ANALYSis}

In this section, we further demonstrate the theoretical results of band-mix selection by carrying out numerical simulations with different utility functions. Moreover, we compare the proposed band-mix selection scheme with the selection of the band with maximum reward, the selection of the band with minimum risk, random based band-mix selection (RAND) and all averaged based band-mix selection (AVR) in terms of spectrum utilization as well as the SUs' satisfactory degree.

\section{A. Verification of the Theoretical Results}

\section{1) Simulation Setup:}

We consider the scenario that the SSP faces the issue how to split one unit traffic in $\mathrm{Mb}$ over his own band and currently unoccupied bands from PSPs by jointly considering the expected reward and the potential risk for OSA. Without loss of generality, we assume the SSP owns one risk-free band, where $R_{s}=8$ and $k=0.4$, and there are two risky bands belonging to the PSPs in range, where $R_{1}=8.2, R_{2}=12, \sigma_{1}=10$, $\sigma_{2}=25$, and $\rho_{12}=-0.4$. The SSP may evaluate different choices of traffic splitting over bands by the utility functions as the follows ${ }^{6}$ :

- Quadratic utility function: $U(R)=a R-\frac{1}{2} b R^{2}$

${ }^{6}$ The expected utility of exponential utility function can be obtained by using $R$ 's moment-generating function, which can be expressed as $E(a-$ $\left.e^{(-b R)}\right)=a-e^{\left(-E(R) b+\frac{\sigma_{R}^{2} b^{2}}{2}\right)}$. The types of utility function are not limited to the two above, e.g., another commonly used function, log utility function, whose expected utility can be approximated using Taylor expansion, is also suitable for this problem.
- Exponential utility function: $U(R)=a-e^{(-b R)}$

For the illustrative purpose, we set $a=1$ and $b=0.25$ and let $R_{s}$ and $R_{i}$ be normally distributed. Leveraging these utility functions, we try to specify the problem we have discussed and verify the derivations as well as the results we have achieved in previous sections by simulations.

2) Results and Analysis:

In Fig. 3(a), the expected utility of the SSP, which is the shape like belt in blue, achieves its maximum approximately at the star point in red on the belt, which represents the optimal traffic splitting over the risk-free band and risky bands. The coordination of the maximum utility is $\left(\sigma_{R}=6.0108, R=\right.$ 9.108, $E(U(R))=15.2389)$ and its mapping on the Expected Reward - Standard Deviation plane is $\left(\sigma_{R}=6.0108, R=\right.$ 9.108). Note that, as shown in Fig. 3(d), the mapping point is on the CSF (in purple) of the band-mix of risk-free band and risky bands, which is tangent to the CSF (i.e., the upper border of the FSS) of the risky band-mixes. The intersecting point on the CSF of the risky bands denotes the optimal proportional composition of the risky bands.

Similar analysis is also suitable for the band-mix selection of the SSP with exponential utility as shown in Fig. 3(b) and Fig. 3(e). It is not surprising to find that although the points of optimal band-mix selection over risk-free band and risky bands with different utility functions are different, these points are all on the CSF of the risk-free band and risky bands on the Expected Reward - Standard Deviation plane. Moreover, once the information of $R_{i}$ is given, the proportional composition of the risky band-mix (i.e., $\omega_{i}$ ) never changes. Both of the two observations are in consistence with our theoretical results. Therefore, following the procedure in 
Sec. III-B, it is not difficult to determine the values of $\omega_{1}$ and $\omega_{2}$. Using corresponding utility function, $\eta$ is easy to obtain as well.

\section{B. Performance Comparison}

In addition, we let the revenue of the SSP be equal to 30, the number of available spectrum bands of PSPs increase from 1 to 6 , and the price of these bands, which is ascending as the risk for OSA increases, be in the range of $(10,30)$. Using exponential utility function, we compare the proposed bandmix selection algorithm for OSA with the maximum reward based band selection, the minimum risk based band selection, RAND and AVR in terms of spectrum utilization and SUs' satisfactory degree.

In Fig. 3(c), we evaluate the performance of spectrum utilization, which is calculated as the ratio of the utilized bands to the total bands. Note that no matter the portion of a band is used by the primary services or used by the secondary services, this portion of spectrum band should be considered as utilized. It is not surprising to see that the performance of the maximum reward based band selection is worst since it always makes the SSP swarm all the traffic on the most risky band from the PSPs for OSA; the performance of the minimum risk based band selection for OSA is much better than the maximum reward based band one, but it is still inferior to the performance of all the band-mix selection schemes for OSA. We find that the proposed band-mix selection outperforms the other two: RAND and AVR. The reason is that the SSP following the proposed band-mix selection scheme will balance the tradeoff between reward and risk for OSA and make the optimal traffic splitting decision. In this way, although the SSP cannot relieve his services from the impact of the primary services' coming back to $100 \%$, he is able to take the better use of the licensed bands from PSPs. By contrast, the spectrum utilization in both RAND and AVR are more likely affected by the activities of the primary services.

Similar analysis also applies to the SUs' satisfactory degree, which is defined to be the ratio of satisfied traffic demands to overall traffic demands of the SUs. Compared with band-mix based selection for OSA, the maximum reward based band selection and the minimum risk based band selection are not good enough. Especially for the maximum reward based one, the performance for OSA degrades with the increase of the number of bands available from PSPs, since there emerge more risky bands, when there are more bands for selection. The satisfactory degree of the proposed band-mix selection for OSA is higher than RAND and AVR when the spectrum bands from PSPs are not abundant for the traffic demands of secondary services as shown in Fig. 3(f).

\section{CONCLUSiON}

We have investigated a reward and risk based band-mix selection for OSA in cognitive radio networks. Instead of gathering all the traffic over one specific band with the maximum reward or minimum risk bought from the PSPs, we enable the SSP to split his traffic over a selected band-mix to alleviate the negative impact of the activities of primary services and create a more stable reward for SSP himself. From the view of the SSP, we define the reward and risk for OSA, formulate the band-mix selection problem, and illustrate the characteristics of the band-mix consisting of correlated spectrum bands at different frequencies. Through the theoretical study, we find: (1) the particular types and the parameters of the SSP's utility function for band-mix selection under our assumptions only determine the ratio of the traffic splitting between the riskfree band of SSP and the risky band-mix from the PSPs; (2) once the information about reward and risk of each band for OSA is given, the optimal proportionate composition of the risky band-mix will be the same for the SSP, no matter what the particular utility function is. By numerical simulations, we further verify these results and show that the performance of the proposed band-mix selection for OSA is better than the selection of the band with maximum reward, the selection of the band with minimum risk, RAND and the AVR in terms of spectrum utilization as well as the SUs' satisfactory degree.

\section{REFERENCES}

[1] FCC, "Spectrum policy task force report," Report of Federal Communications Commission, Et docket No. 02-135, November 2002.

[2] D. Grandblaise, P. Bag, P. Levine, K. Moessner, and M. Pan, "Microeconomics inspired mechanisms to manage dynamic spectrum allocation," in Proc. of IEEE International Symposium on New Frontiers in Dynamic Spectrum Access Networks, DySPAN '07, Dublin, Ireland, April 2007.

[3] S. Sengupta and M. Chatterjee, "An economic framework for dynamic spectrum access and service pricing," IEEE/ACM Transactions on Networking, vol. 17, no. 4, pp. 1200-1213, Aug. 2009.

[4] B. Wang, Z. Han, and K. J. R. Liu, "Distributed relay selection and power control for multiuser cooperative communication networks using buyer/seller game," in Proc. of IEEE Conference on Computer Communications, INFOCOM 2007, Anchorage, AK, May 2007.

[5] M. Pan, F. Chen, X. Yin, and Y. Fang, "Fair profit allocation in the spectrum auction using the shapley value," in Proc. of IEEE Global telecommunications conference, Globecom '09, Honolulu, HI, USA, December 2009

[6] X. Zhou, S. Gandhi, S. Suri, and H. Zheng, "ebay in the sky: strategyproof wireless spectrum auctions," in Proc. of Mobile Computing and Networking, Mobicom '08, San Francisco, CA, September 2008.

[7] J. Jia, Q. Zhang, Q. Zhang, and M. Liu, "Revenue generation for truthful spectrum auction in dynamic spectrum access," in Proc. of ACM International Symposium on Mobile Ad Hoc Networking and Computing, ACM MobiHoc, 2009, New Orleans, LA, May 2009.

[8] Y. Xing, R. Chandramouli, and C. Cordeiro, "Price dynamics in competitive agile spectrum access markets," IEEE Journal on Selected Areas in Communications, vol. 25, no. 3, pp. 613-621, April 2007.

[9] D. Niyato and E. Hossain, "Competitive pricing for spectrum sharing in cognitive radio networks: Dynamic game, inefficiency of nash equilibrium, and collusion," IEEE Journal on Selected Areas in Communications, vol. 26, no. 1, pp. 192-202, January 2008.

[10] A. Motamedi and A. Bahai, "Optimal channel selection for spectrumagile low-power wireless packet switched networks in unlicensed band," EURASIP J. Wirel. Commun. Netw., vol. 2008, pp. 1-10, 2008.

[11] H. Markowitz, "Portfolio selection," The Journal of Finance, vol. 7 , no. 1, pp. 77-91, Mar. 1952.

[12] L. Giupponi, R. Agusti, J. Perez-Romero, and O. S. Roig, "A novel approach for joint radio resource management based on fuzzy neural methodology," IEEE Transactions on Vehicular Technology, vol. 57, no. 3, pp. 1789-1805, May 2008.

[13] D. Chen, S. Yin, Q. Zhang, M. Liu, and S. Li, "Mining spectrum usage data: a large-scale spectrum measurement study," in Proc. of international conference on Mobile computing and networking, ACM Mobicom, 2009, Beijing, China, September 2009. 